\title{
Presença de deficiência auditiva e hipertensão em adultos e idosos
}

\author{
Aline Ferreira Vieira*; Isabela Hoffmeister Menegotto**; Adriane Ribeiro Teixeira**; \\ Luiza Fernandes Millão****
}

\section{Resumo}

Este estudo teve como objetivo verificar a possível relação entre a presença de hipertensão arterial sistêmica (HAS) e deficiência auditiva (DA) em adultos e idosos, buscando também analisar a influência das variáveis gênero e idade em ambos as alterações. Foram analisados os resultados das triagens auditivas e avaliações de hipertensão arterial que se encontravam em prontuários dos cursos de Fonoaudiologia e Enfermagem, respectivamente. Avaliaram-se 76 indivíduos, sendo 55 do sexo feminino e 21 do masculino, com idades compreendidas entre 47 e 80 anos. A pesquisa apontou que idosos apresentam uma maior prevalência de triagens auditivas alteradas. Não houve associação significativa entre triagem auditiva/HAS e gênero. A idade não foi determinante na presença ou ausência de HAS e não houve associação entre presença de HAS e falha na triagem auditiva. Os indivíduos hipertensos não apresentaram maior falha na triagem auditiva do que os normotensos. A HAS não foi relacionada estatisticamente à terceira idade, porém com o avanço da idade apareceram mais alterações auditivas, provavelmente em razão do processo de enveIhecimento.

Palavras-chave: Hipertensão. Audição. Idoso.

\section{Introdução}

A audição desempenha um importante papel na comunicação humana, por ser fundamental na inserção do sujeito no meio em que vive. A deficiência auditiva proporciona inúmeros danos à qualidade de vida dos indivíduos portadores, trazendo prejuízos que vão desde a não participação na vida familiar até o isolamento e depressão.

$\mathrm{O}$ indivíduo portador de deficiência auditiva necessita de intervenção especializada e, quanto mais precoce for

* Acadêmica do curso de Fonoaudiologia da Universidade Luterana do Brasil. Endereço para correspondência: Aline Ferreira Vieira, Rua Viena 108, Bairro Niterói, CEP 92130-280, Canoas - RS. E-mail: linex_b@hotmail. com

** Fonoaudióloga. Doutora em Distúrbios da Comunicação Humana pela Universidade Federal de São Paulo. Professora do curso de Fonoaudiologia da Universidade Luterana do Brasil.

**** Fonoaudióloga. Doutora em Gerontologia Biomédica pela Pontifícia Universidade Católica do Rio Grande do Sul.

***** Enfermeira. Professora do curso de Enfermagem da Universidade Luterana do Brasil.

$\hookrightarrow$ Recebido em janeiro de 2009 - Avaliado em março de 2009.

$\hookrightarrow$ doi:10.5335/rbceh.2009.023 
realizado o diagnóstico, mais rapidamente ele terá assistência necessária em termos de reabilitação e orientação.

Tratando-se de doenças crônicas, sabe-se que, em geral, a idade avançada está associada a um comprometimento maior dos sentidos. Dentre as alterações sensoriais que acompanham o processo do envelhecimento, a deficiência auditiva ou diminuição da função auditiva, conhecida como "presbiacusia", é uma das mais incapacitantes. (MARQUES; KOZLOWSKI; MARQUES, 2004).

A deficiência auditiva torna a percepção dos sons consoantes muito difícil, especialmente quando a comunicação ocorre em ambientes ruidosos. Idosos portadores de presbiacusia experimentam diminuição da sensibilidade auditiva e redução da inteligibilidade da fala, o compromete a comunicação verbal. Muitas vezes respostas inadequadas de indivíduos idosos presbiacústicos geram uma imagem de senilidade, a qual pode não condizer com a realidade. (RUSSO, 2004).

Sabe-se que a deficiência auditiva é desencadeada por vários fatores. As alterações metabólicas e vasculares podem afetar a audição de muitas maneiras, com diferentes tipos de queixas relacionados à deficiência auditiva.

A resistência que o sangue encontra nas artérias periféricas versus o volume de sangue que o coração bombeia a cada batida chama-se "pressão arterial". Quando esta pressão apresenta níveis elevados, associada a alterações metabólicas, hormonais e genéticas, dentre outras, caracteriza-se um quadro de hipertensão arterial sistêmica. (LIPP; ROCHA, 1996; LUNA, 1989).
O diagnóstico de hipertensão arterial é definido quando os valores de pressão arterial sistólica são iguais ou superiores a $140 \mathrm{mmHg}$ e pressão arterial diastólica iguais ou superiores a $90 \mathrm{mmHg}$. $\mathrm{Na}$ população idosa se consideram para pressão arterial sistólica alterada valores iguais ou superiores a $160 \mathrm{mmHg}$. (SMELTZER; BARE, 1998).

De acordo com Weinstein (1999), artrite, hipertensão arterial e perda auditiva são os três problemas crônicos mais frequentemente encontrados em idosos. A hipertensão arterial, por sua vez, na população idosa também pode afetar o adequado funcionamento do sistema auditivo.

A pressão arterial elevada, segundo Marchiori, Freitas e Vieira (2002), pode provocar desde a involução gradual dos vasos da orelha interna até hemorragias na orelha. Ocorre uma perda do ligamento espiral da cóclea, que audiometricamente se caracteriza por perda auditiva nas frequências altas ou presbiacusia.

Os tecidos da orelha interna são ativos metabolicamente, o que leva a que uma privação metabólica, mesmo que por um curto período, possa resultar em perda auditiva. (ZIMMERMAN, 1999).

Segundo Torres, Figueiredo e Waltermann (2006), o indivíduo portador de hipertensão arterial necessita de uma abordagem multidisciplinar por se tratar de uma doença multifatorial. A presença do fonoaudiólogo na equipe multiprofissional envolvida na hipertensão arterial é de grande importância, porque esta é fator de risco para deficiência auditiva (BARALDI; ALMEIDA; BORGES, 2004; MARCHIORI; FREITAS; VIEIRA, 2002; MARCHIORI; REGO; MATSUO, 2006), 
muitas vezes confundida com comportamento senil do idoso por aqueles que convivem com ele. Assim, a fim de prevenir e detectar as alterações provocadas pelo envelhecimento seria fundamental que indivíduos portadores de hipertensão arterial realizassem também uma avaliação audiológica.

Dessa forma, o objetivo do presente estudo foi verificar os resultados das triagens auditivas de adultos e idosos hipertensos e não hipertensos na tentativa de buscar uma correlação entre os dois fatores.

\section{Metodologia}

A presente pesquisa inseriu-se dentro do projeto intitulado "Caracterização de idosos" atendidos em projeto extensionista da FGA Adriane Teixeira, aprovado pelo Comitê de Ética em Pesquisa conforme protocolo $\mathrm{n}^{\mathrm{0}} 125$ h - 2008. A pesquisa foi realizada por meio da análise de dados secundários, ou seja, foram analisados os resultados das triagens auditivas e avaliações de hipertensão arterial que se encontram, respectivamente, em prontuários dos cursos de Fonoaudiologia e Enfermagem da Universidade Luterana do Brasil.

Embora esta pesquisa tenha sido realizada utilizando dados secundários, foi assumida, não só pela equipe responsável pelos prontuários como pela examinadora e orientadora, a garantia de confidencialidade e anonimato dos pacientes. Assim, a equipe responsável pelos prontuários, a examinadora e orientadora da pesquisa receberam e assinaram o termo de compromisso de utilização de dados.
A coleta da base de dados foi realizada em junho de 2007 por alunos bolsistas dos cursos de Fonoaudiologia e Enfermagem, supervisionada pelos professores dos respectivos cursos. Foi feita a seleção de uma sala silenciosa no prédio 55 (Ulbra) por meio de um decibelímetro Minipa MLS 1351-S, para realização das triagens auditivas (meatoscopia e pesquisa dos limiares auditivos). As avaliações da hipertensão arterial (medição da pressão arterial e pergunta sobre presença de hipertensão) foram feitas em outra sala no mesmo prédio.

Como o ambiente onde foi realizada a triagem auditiva não era isolado acusticamente, foram excluídos da pesquisa os valores de limiar obtidos na frequência de $500 \mathrm{~Hz}$. Assim, foram considerados indivíduos com triagens auditivas normais aqueles que apresentaram limiares auditivos até $25 \mathrm{~dB}$ e com triagens auditivas alteradas aqueles que tiveram limiares auditivos acima de $25 \mathrm{~dB}$. Para posterior análise dos limiares auditivos foi utilizada a classificação da Organização Mundial da Saúde (1997).

$\mathrm{Na}$ análise dos prontuários da enfermagem foram considerados como portadores de pressão arterial normal os indivíduos adultos com pressão arterial até 130/85 mmHg e idosos com pressão arterial até 150/85 mmHg. Foram considerados portadores de HAS os indivíduos adultos com pressão arterial igual ou superior a 140/90 mmHg e idosos com pressão arterial igual ou superior 160/90 mmHg. (SMELTZER; BARE, 1998), de acordo com Ministério da Saúde.

Para obtenção dos dados foi elaborada uma ficha de triagem auditiva contendo as informações cruciais para 
a pesquisa em questão, como nome, número do prontuário, idade, sexo, audição (OD e OE) e presença ou ausência de hipertensão. Assim, obteve-se uma amostra total de 76 indivíduos, sendo $55(72 \%)$ do sexo feminino e $21(27 \%)$ do sexo masculino, com idades compreendidas entre 47 e 80 anos.

A partir disso foi elaborada uma tabela para análise estatística dos dados obtidos, contendo as informações coletadas nos prontuários, para verificar a presença de perda auditiva em indivíduos hipertensos e não hipertensos, analisando também as variáveis idade e gênero nos distúrbios pesquisados.

\section{Resultados}

De acordo com o objetivo deste estudo, os dados obtidos dos dois grupos estudados - indivíduos hipertensos e indivíduos não hipertensos - foram comparados segundo a presença ou não de perda auditiva, o sexo e a idade. Esses dados foram transformados em valores numéricos e a correlação entre os distúrbios pesquisados e suas variáveis foi testada por meio do teste qui-quadrado. O nível de significância utilizado para as comparações estatísticas foi de $0,05(5 \%)$.

Dos 76 indivíduos avaliados, obtiveram-se vinte adultos com idades variando entre 49 e 59 anos e 56 idosos com idades variando entre 60 e 80 anos. Do total de indivíduos, 55 eram do sexo feminino e 21, do sexo masculino.

A Tabela 1 mostra a associação entre o resultado da triagem auditiva e a idade. Verificam-se na Tabela $14(37,8 \%)$ adultos e $23(62,2 \%)$ idosos com triagem auditiva alterada e seis $(15,4 \%)$ adultos e $33(84,6 \%)$ com triagem auditiva normal. A associação significativa entre essas variáveis demonstrada pelo teste qui-quadrado $(p=0,026)$ aponta que os indivíduos idosos apresentam uma maior prevalência de triagens alteradas, o que sugere a presença de alterações auditivas, do que os indivíduos adultos.

Tabela 1 - Frequências percentuais e absolutas de triagens auditivas normais e alteradas em indivíduos adultos e idosos na amostra do presente estudo.

\begin{tabular}{|c|c|c|c|c|c|c|}
\hline \multirow{3}{*}{ Idade } & \multicolumn{4}{|c|}{ Triagem auditiva } & \multirow{2}{*}{\multicolumn{2}{|c|}{ Total }} \\
\hline & \multicolumn{2}{|c|}{ Normal } & \multicolumn{2}{|c|}{ Alterada } & & \\
\hline & n. & $\%$ & $\mathrm{n}$. & $\%$ & $\mathrm{n}$. & $\%$ \\
\hline Adulto & 14 & 37,8 & 6 & 15,4 & 20 & 26,3 \\
\hline Idoso & 23 & 62,2 & 33 & 84,6 & 56 & 73,7 \\
\hline Total & 37 & 100,0 & 39 & 100,0 & 76 & 100,0 \\
\hline
\end{tabular}

Teste qui-quadrado $\mathrm{p}=0,026^{*}$.

A Tabela 2 apresenta a associação entre o resultado da triagem auditiva e sexo. Em relação ao sexo feminino, das 55 mulheres, $30(81 \%)$ apresentaram triagem auditiva normal e $25(64,1 \%)$, triagem auditiva alterada. Já os indivíduos do sexo masculino, sete $(18,9 \%)$ apresentaram triagem auditiva normal e $14(35,9 \%)$, alterada. Embora não tenha existido uma associação significativa entre estas variáveis $(\mathrm{p}=0,098)$, observa-se no grupo avaliado uma tendência de as mulheres apresentarem mais alterações auditivas do que os homens. 
Tabela 2 - Frequências porcentuais e absolutas de triagens auditivas normais e alteradas em indivíduos do sexo masculino e do sexo feminino.

\begin{tabular}{|c|c|c|c|c|c|c|}
\hline \multirow{3}{*}{ Idade } & \multicolumn{4}{|c|}{ Triagem auditiva } & \multirow{2}{*}{\multicolumn{2}{|c|}{ Total }} \\
\hline & \multicolumn{2}{|c|}{ Normal } & \multicolumn{2}{|c|}{ Alterada } & & \\
\hline & $\mathrm{n}$. & $\%$ & $\mathrm{n}$. & $\%$ & $\mathrm{n}$. & $\%$ \\
\hline Adulto & 30 & 81,1 & 25 & 64,1 & 55 & 72,4 \\
\hline Idoso & 7 & 18,9 & 14 & 35,9 & 21 & 27,6 \\
\hline Total & 37 & 100,0 & 39 & 100,0 & 76 & 100,0 \\
\hline
\end{tabular}

Teste qui-quadrado $\mathrm{p}=0,098$.

A Tabela 3 apresenta a associação entre HAS e idade. Dos vinte adultos avaliados, apenas seis $(22,2 \%)$ foram considerados hipertensos e $14(28,6 \%)$, não hipertensos. Em relação aos indivíduos idosos, dos 56 participantes da amostra, $21(77,8 \%)$ eram hipertensos e 35 (71,4\%), não hipertensos. A ausência de associação significativa entre essas variáveis, demonstrada pelo teste qui-quadrado ( $\mathrm{p}=0,547$ ), aponta que a idade não foi determinante na presença ou ausência de hipertensão na amostra estudada.

Tabela 3 - Frequências porcentuais e absolutas de presença ou ausência de hipertensão arterial em indivíduos adultos e idosos.

\begin{tabular}{|c|c|c|c|c|c|c|}
\hline \multirow{3}{*}{ Idade } & \multicolumn{4}{|c|}{ Triagem auditiva } & \multirow{2}{*}{\multicolumn{2}{|c|}{ Total }} \\
\hline & \multicolumn{2}{|c|}{ Normal } & \multicolumn{2}{|c|}{ Alterada } & & \\
\hline & $\mathrm{n}$. & $\%$ & $\mathrm{n}$. & $\%$ & $\mathrm{n}$. & $\%$ \\
\hline Adulto & 14 & 28,6 & 6 & 22,2 & 20 & 26,3 \\
\hline Idoso & 35 & 71,4 & 21 & 77,8 & 56 & 73,7 \\
\hline Total & 49 & 100,0 & 27 & 100,0 & 76 & 100,0 \\
\hline
\end{tabular}

Teste qui-quadrado $\mathrm{p}=0,547$.

A Tabela 4 apresenta associação entre HAS e sexo. Das 55 mulheres, 21 $(77,8 \%)$ apresentaram hipertensão, e 34 $(69,4 \%)$, não. Dos 21 homens, apenas seis
$(22,2 \%)$ apresentaram hipertensão arterial. Mais uma vez não houve associação significativa demonstrada entre estas variáveis $(\mathrm{p}=0,434)$.

Tabela 4 - Frequências porcentuais e absolutas de presença ou ausência de hipertensão arterial em indivíduos do sexo feminino e do sexo masculino.

\begin{tabular}{|c|c|c|c|c|c|c|}
\hline \multirow{3}{*}{ Idade } & \multicolumn{4}{|c|}{ Triagem auditiva } & \multirow{2}{*}{\multicolumn{2}{|c|}{ Total }} \\
\hline & \multicolumn{2}{|c|}{ Normal } & \multicolumn{2}{|c|}{ Alterada } & & \\
\hline & $\mathrm{n}$. & $\%$ & $\mathrm{n}$. & $\%$ & $\mathrm{n}$. & $\%$ \\
\hline Adulto & 34 & 69,4 & 21 & 77,8 & 55 & 72,4 \\
\hline Idoso & 15 & 30,6 & 6 & 22,2 & 21 & 27,6 \\
\hline Total & 49 & 100,0 & 27 & 100,0 & 76 & 100,0 \\
\hline
\end{tabular}

Teste qui-quadrado $\mathrm{p}=0,434$. 
A Tabela 5 apresenta o resultado da comparação entre a presença de hipertensão arterial e falha na triagem auditiva. Verifica-se que $48,7 \%$ indivíduos apresentaram triagem auditiva normal, dos quais $37 \%$ eram hipertensos e $55,1 \%$, não hipertensos. Dos indivíduos que tiveram triagem auditiva alterada
(51,3\%), $63 \%$ eram hipertensos e 44,9\%, não hipertensos. A análise estatística demonstrou que não houve associação significativa entre triagem auditiva e hipertensão arterial $(p=0,132)$, ou seja, os indivíduos hipertensos não apresentaram mais falhas na triagem auditiva que os indivíduos normotensos.

Tabela 5 - Frequências porcentuais e absolutas de triagens auditivas normais ou alteradas e presença ou ausência de hipertensão arterial.

\begin{tabular}{|c|c|c|c|c|c|c|}
\hline \multirow{3}{*}{ Idade } & \multicolumn{4}{|c|}{ Triagem auditiva } & \multirow{2}{*}{\multicolumn{2}{|c|}{ Total }} \\
\hline & \multicolumn{2}{|c|}{ Normal } & \multicolumn{2}{|c|}{ Alterada } & & \\
\hline & $\mathrm{n}$. & $\%$ & $\mathrm{n}$. & $\%$ & $\mathrm{n}$. & $\%$ \\
\hline Adulto & 27 & 55,1 & 10 & 37,0 & 37 & 48,7 \\
\hline Idoso & 22 & 44,9 & 17 & 63,0 & 39 & 51,3 \\
\hline Total & 49 & 100,0 & 27 & 100,0 & 76 & 100,0 \\
\hline
\end{tabular}

Teste qui-quadrado $\mathrm{p}=0,132$.

\section{Discussão}

No Brasil a HAS é um dos mais importantes fatores de risco cardiovascular. Estima-se que metade dos óbitos em pacientes acima de cinquenta anos se deva a doenças cardiovasculares, sendo $80 \%$ delas de portadores de HAS. (TOSCANO-BARBOSA, 2007). Por sua vez, a deficiência auditiva tem um efeito adverso no estado funcional, na qualidade de vida, na função cognitiva e no bem-estar emocional, comportamental e social do indivíduo. Alguns estudos demonstram prevalência de $63 \%$ de deficiência auditiva em indivíduos idosos A associação entre a hipertensão arterial e deficiência auditiva é amplamente estudada na literatura, sendo os achados controversos. (BARALDI; ALMEIDA; BORGES, 2004).

No presente estudo observa-se na Tabela 1 que os indivíduos idosos apre- sentam maior número de triagens auditivas alteradas que os indivíduos adultos, o que concorda com a alta prevalência de alterações auditivas na terceira idade citada por outros estudos. (MARCHIORI; FREITAS; VIEIRA, 2002; FUESS; CERCHIARI, 2003; MARCHIORI; REGO; MATSUO, 2006; BARALDI; ALMEIDA; BORGES, 2007). Isso se explica pelo fato de o processo de envelhecimento ser global e deteriorativo, ou seja, com a idade há uma instalação progressiva de alterações estruturais em todo o organismo, inclusive no sistema auditivo.

Quanto à variável sexo, neste estudo verificou-se que, embora não tenha ocorrido associação significativa (Tab. 2), as mulheres apresentaram mais alterações auditivas do que os homens. Este dado discorda da literatura pesquisada, a qual relata que os indivíduos do sexo masculino apresentam um declínio da acuidade auditiva com a idade, como 
fator de risco independente para perda auditiva (PEARSON et al., 1995; COLLET et al., 1992; FUESS; CERCHIARI, 2003; BARALDI; ALMEIDA; BORGES, 2007). Este resultado, no presente estudo, pode ter sido influenciado pelo fato de a amostra ser composta por um número maior de indivíduos do sexo feminino do que do sexo masculino.

Ainda neste estudo, a idade (Tab. 3) não foi determinante na presença ou ausência de hipertensão arterial, embora esta se trate de uma doença crônica que é mais frequentemente encontrada na população idosa. (BONADESE, 1998; WEINSTEIN 1999). Assim, este achado não corrobora outros estudos com indivíduos hipertensos e normotensos (BONADESE, 1998; ZAITUNE et. al., 2006; BARBOSA; TOSCANO, 2007), mostrando que na amostra estudada não há uma prevalência maior estatisticamente significativa de hipertensão na terceira idade.

Observou-se que a presença de hipertensão nos indivíduos do sexo feminino e masculino foi semelhante (Tab. 4). Este dado discorda dos achados obtidos em outros estudos (OPARIL, 2005; ZAITU$\mathrm{NE}$ et. al., 2006), nos quais a prevalência de hipertensão foi mais elevada nos indivíduos do sexo feminino. Os autores justificam o ocorrido com o argumento de que as mulheres apresentam maior tendência para o autocuidado e buscam, assim, mais assistência médica do que os homens, o que tenderia a aumentar a probabilidade de terem a hipertensão diagnosticada.

Este estudo não demonstrou existência de associação significativa entre hipertensão arterial e falha na triagem auditiva (Tab. 5). Os achados discordam de outros estudos (MARCHIORI; FREITAS; VIEIRA, 2002; MARCHIORI; REGO, MATSUO, 2006), nos quais foi verificado um considerável aumento do número de perdas auditivas em indivíduos hipertensos. Porém, os presentes resultados corroboram os achados dos estudos de Fuess e Cerchiari (2003) e Baraldi, Almeida e Borges (2004), que também não observaram tal associação.

Acredita-se que a amostra do estudo em questão possa estar diretamente relacionada com a não existência de associação entre hipertensão e falha na triagem auditiva. Comparando com a literatura pesquisada, verificou-se que a ocorrência de associação existiu nos estudos em que a amostra foi composta por um número maior de indivíduos (552 e 308, respectivamente, nos estudos de MARCHIORI; FREITAS; VIEIRA, 2002; MARCHIORI, REGO, MATSUO, 2006), diferentemente dos demais estudos, nos quais a amostra foi menor (114 e 70, respectivamente, em FUESS; CERCHIARI, 2003; BARALDI; ALMEIDA; BORGES 2004), e não houve associação significativa entre os distúrbios pesquisados.

Outro fator que pode ter influenciado este resultado foi o fato de a análise ser feita somente com a melhor orelha e não se terem incluídos os valores obtidos nas frequências de 6000 e $8000 \mathrm{hz}$.

A hipertensão pode não estar diretamente relacionada com a presença de alterações auditivas, mas associada à idade, e por se tratar de uma doença multifatorial, que provoca inúmeras alterações em todo nosso organismo, pode 
agir como um fator somador na deterioração do sistema auditivo.

Assim, os indivíduos hipertensos podem ser considerados uma população de risco para alterações auditivas. Esse fato torna necessário averiguar, sempre que possível, a audição desses indivíduos, pois, quanto mais precoce for feito o diagnóstico, mais o indivíduo terá assistência necessária em termos de reabilitação e orientação.

\section{Conclusão}

Analisando os resultados obtidos na presente pesquisa e relacionando-os entre si, pode-se concluir que com o avanço da idade aparecem mais alterações auditivas, provavelmente em razão do processo global de envelhecimento do organismo. Já em relação aos problemas circulatórios, a idade não foi fator determinante no presente estudo, mostrando que a HAS não foi relacionada estatisticamente à terceira idade.

Em relação à variável gênero, os dados obtidos discordaram dos achados na literatura pesquisada. A falha na triagem auditiva teve maior ocorrência nas mulheres e a presença de HAS foi semelhante em ambos os sexos.

Neste estudo, os indivíduos hipertensos não apresentaram maior falha na triagem auditiva do que os normotensos, o que discorda de alguns estudos, mas concorda com outros, sugerindo a necessidade de mais pesquisas sobre a relação entre HAS e PA, com maior número de indivíduos, pois os achados ainda são controversos.

Em virtude disso, reforça-se a importância de os profissionais da área da saúde solicitarem, sempre que possível, a avaliação audiológica aos indivíduos hipertensos. O diagnóstico precoce pode propiciar uma melhor qualidade de vida, evitando que acabem se isolando ou sendo isolados pela comunidade em razão das suas dificuldades auditivas.

\section{Presence of hearing loss and hypertension in adults and elderly}

\section{Abstract}

This study had as objective to verify the possible relationship between the presence of systemic blood hypertension $(\mathrm{SBH})$ and hearing loss $(\mathrm{HL})$ in adults and elderly, also looking for to analyze the influences of gender and age variables in both alterations. It was analyzed the results of the hearing screening and arterial hypertension evaluations that were found in handbooks of the courses of Speech and hearing therapy and Nursing respectively. It was evaluated 76 subjects, being 55 female and 21 male, with ages between 47 and 80 years old. The research pointed that elderly present a larger prevalence of altered hearing screening. There was no significant association between hearing screening/SBH and gender. The age was not decisive in the presence or absence of $\mathrm{SBH}$ and there was no association between the presence of $\mathrm{SBH}$ and failure in the hearing screening. The hypertensive subjects didn't present larger failure in the hearing screening than the normotensives. The SBH was not statistically related to elderly citizen, however with age advancing, it appeared more hearing alterations, probably because of the aging process.

Key words: Hypertension. Audition. Elderly 


\section{Referências}

BARALDI, G. D.; ALMEIDA, L. C.; BORGES, A. C. L. C. Perda auditiva e hipertensão: achados em um grupo de idosos. Revista Brasileira de Otorrinolaringologia, São Paulo, v. 70, n. 5, p. 640-644, 2004.

BARALDI, G. D.; ALMEIDA, L. C.; BORGES, A. C .L. C. Evolução da perda auditiva no decorrer do envelhecimento. Revista Brasileira de Otorrinolaringologia, xx, v. 73, n. 1, p. 64-70, 2007.

BODANESE, L. C. Hipertensão arterial no idoso. Revista Brasileira de Clínica e Terapêutica, São Paulo, v. 24, n. 5, p. 194-199, 1998.

COLLET, L. et al. Auditory brainstem reponse (ABR) latency: relative importance of age, sex and sensorineural hearing-loss using a mathematical model of the audiogram. International Journal Neuroscience, v. 67, n. 4, p. 187-97, 1992.

FUESS, V. L. R.; CERCHIARI, D. P. Estudo da hipertensão arterial sistêmica e do diabetes mellitus como fatores agravantes da presbiacusia. Arquivos Internacionais de Otorrinolaringologia, São Paulo, v. 7, n. 2, p. 229-232, 2003.

LIPP, M. N.; ROCHA, J. C. Stress, HAS e qualidade de vida: um guia de tratamento para o hipertenso. 2. ed. Campinas: Papirus, 1996.

LUNA, R. L. Hipertensão arterial. Rio de Janeiro: Medsi, 1989.

MARCHIORI, L. L. M.; FREITAS, S. V.; VIEIRA, M. Análise de prevalência das queixas e perdas auditivas de pacientes com hipertensão arterial submetidos à avaliação audiológica. Pancast. Fono. Atual., São Paulo v. 21, n. 2, p. 97-104, 2002.

MARCHIORI, L. L. M.; REGO, E. E.; MATSUO, T. Hipertensão como fator associado à perda auditiva. Revista Brasileira de Otorrinolaringologia, São Paulo, v. 72, n. 4, p. 533-40, 2006.
MARQUES, A. C. O.; KOZLOWSKI, L.; MARQUES, J. M. Reabilitação auditiva no idoso. Revista Brasileira de Otorrinolaringologia, São Paulo, v. 70, n. 6, p. 806-11, 2004.

OPARIL, S. Hipertensão arterial. In: GOLDMAN, L.; AUSIELLO, D. (Org.). Cecil: tratado de medicina interna. Trad. de Ana Kemper et al. Rio do Janeiro: Elsevier, 2005. p. 289-292.

PEARSON, J. D. et al. Gender differences in a longitudinal study of age-associated hearing loss. The Journal of the Acoustical Society of America, v. 97, n. 2, p. 1196-1205, 1995.

RUSSO, I. P. Intervenção fonoaudiológica na terceira idade. Rio de Janeiro: Revinter, 2004.

SMELTZER, S. C.; BARE, B. Brunner \& Suddarth: tratado de enfermagem médicocirúrgica. Trad. de Carolina S. Motta et al. 8. ed. Rio de Janeiro: Guanabara Koogan, 1998. v. 2

TORRES, L. S.; FIGUEIREDO, M. R. B.; WALTERMANN, M. Hipertensão arterial: um desafio para a equipe multiprofissional. Logos, Canoas, v. 17, n. 1, p. 129-138, jan./ jun. 2006.

TOSCANO-BARBOSA, E. Expectativas para abordagem da hipertensão no Brasil, com a mudança do século. Revista Brasileira de Hipertensão, Rio de Janeiro, v. 7, n. 1, p. 7-10, 2007.

WEINSTEIN, B. E. Presbiacusia. In: KATZ, J. (Org.). Tratado de audiologia clínica. 4. ed. São Paulo: Manole, 1999. p. 562-583.

ZAITUNE, M. P. A. et al. Hipertensão arterial em idosos: prevalência, fatores associados e práticas de controle no Município de Campinas, São Paulo, Brasil. Caderno de Saúde Pública, São Paulo, v. 22, n. 2, p. 285-194, 2006.

ZIMMERMAN, R. L. Considerações neurológicas em audiologia. In: KATZ, J. (Org.). Tratado de audiologia clínica. 4. ed. São Paulo: Manole, 1999. p. 32-56. 